\title{
Identification of AC003986.3 as an Independent Risk Factor for Glioma Patient Survival and Functional Analysis
}

Qinglin Liu

Beijing Tiantan Hospital

Huijian Ge

Beijing Tiantan Hospital

Peng Jiang ( $\square$ ttyyjp@126.com )

Beijing Tiantan Hospital https://orcid.org/0000-0002-5714-3411

Research article

Keywords: glioma, IncRNA, metabolism, patient survival, risk factor

Posted Date: July 24th, 2020

DOI: https://doi.org/10.21203/rs.3.rs-44898/v1

License: (c) (i) This work is licensed under a Creative Commons Attribution 4.0 International License.

Read Full License 


\section{Abstract}

Background: To validate the potential of AC003986.3 in predicting glioma patient survival and analyze its underlying mechanism.

Methods: Gene expression and clinical features of the patients with gliomas were obtained from The Cancer Genome Atlatls. Correlation between AC003986.3 expression profile and patient clinical features and survival were analyzed. Multivariate Cox regression was employed to determine the risk factors for patient survival and construct the prediction model for survival. Validation of the multivariate Cox regression model was performed by comparing the survival curves between the model-predicted high and low death risk subgroups and calculating the accuracy of predicting $1,2,3$, and 5 years survival by the model. Target genes were predicted with Ensemble Browser. Gene set enrichment analysis was performed to explore AC003986.3 related gene sets enrichment in Gene Ontology and Kyoto Encyclopedia of Genes and Genomes pathways.

Results: 655 samples with gene expression and complete clinical features were obtained from The Cancer Genome Atlas. Clinical features enrolled in this study were follow up time, survival status, race, gender, race and pathological grade. AC003986.3 expression was positively related to patient age and pathological grade. Patients with High AC003986.3 expression suffered a poorer survival than those with low expression. Multivariate Cox regression revealed that AC003986.3 expression was an independent risk factor for patient survival irrespective of age and pathological grade. Predicted by Ensemble Browser, TWIST1 was identified as the target of AC003986.3. Gene set enrichment analysis revealed that AC003986.3 related gene sets were mainly enriched in cell metabolism.

Conclusions: AC003986.3 expression was closely related to age and pathological grade in glioma patients, and was an independent risk factor for patient survival irrespective of age and pathological grade. AC003986.3 was mainly involved in regulating tumor cell metabolism, and this effect is probably mediated by TWIST1.

\section{Introduction}

Glioma is featured by increased incidence with patient age and accounts for $75 \%$ of all primary brain malignancies in adults, [1] of which glioblastoma is the most aggressive pathological type. Gliomas are highly heterogeneous, with the 5-year survival rate varies greatly between pathological grades from $5.5 \%$ for glioblastoma to $94 \%$ for pilocytic astrocytoma.[1, 2] Progress in genetic studies for tumorigenesis has direct the transmission from light microscope-based traditional classification to the molecular parameters incorporated classifications - the 2016 CNS WHO.[3] Although the great challenges in nomenclature, nosology and reporting structure, this new classification is more objective and precise to allow for improved tailoring of patient therapy.[3] The 2016 CNS WHO represents the trend from pathological classification to molecular classification for this highly heterogeneous tumor. 
Besides the traditional surgical resection, chemotherapy, and radiotherapy, many new treatment modalities such as immunotherapy, gene therapy, and gene therapy are in clinical trials. [3] However, effective therapies for gliomas are still limited. Redundancy and complexity of intracellular signaling pathways and tumor molecular heterogeneity are partially responsible for this frustrating results.[3] Thus, mapping the gene network and identifying the key drivers for tumorigenesis would benefit the diagnosis, classification and effective targeted treatment for this heterogeneous disease.

Besides the mutations that have been adopted by the 2016 CNS WHO, such as $1 p 19 q$ codeletion, IDH1/2 mutations, and MGMT promoter methylation, [3] various gene mutations or aberrant expression related to tumorigenesis and patient prognosis have been reported.[4-6] Although the functional studies of a single gene may not be enough to change the ongoing treatment strategies for gliomas, the accumulation of these findings would certainly drive a revolution in the future. Therefore, screening and functional analysis of a single gene with treatment potentials are still work of significance.

Began in 2006, The Cancer Genome Atlas (TCGA) program (https://www.cancer.gov) has molecularly characterized over 20,000 primary cancer and matched normal samples spanning 33 cancer types. The publically available genomic, epigenetic, transcriptomic, and proteomic data in TCGA has made it an ideal treasury to explore the underlying mechanisms for tumorigenesis and screen the potential treatment target.[7]

In this study, we performed data mining with the glioma datasets in TCGA to explore the expression and clinical significance of a novel IncRNA named AC003986.3, and gene set enrichment analysis (GSEA) was performed to predict the biological functions of this gene.

\section{Materials And Methods}

Data acquisition

Gene expression and clinical data of the low-grade glioma and glioblastoma datasets were obtained for TCGA. 696 samples were contained in these two datasets. Clinical features and the gene expression profiles were extracted with a perl script. The clinical information enrolled in this study were follow up time, survival status, grade, age, gender, and race. Samples without complete clinical information were excluded. Finally, a total of 655 patients with complete clinical information and gene expression profiles were included in this study. As all the data were publicly accessible, informed consent was not required from the patients. The study was approved by the institutional ethics committee of Beijing Tiantan Hospital, Capital University of China.

Correlations between gene expression and clinical features

AC003986.3 expression was performed log2 transformation. For continuous variate age, linear regression was performed to explore the correlation of gene expression profile to patient age. For the categorical 
clinical features such as gender, race and tumor grade, AC003986.3 gene expression was compared between the subgroups.

Correlations between gene expression and patient survival

Univariate Cox regression was performed to explore the correlation between AC003986.3 expression and patient survival. Then, all the patients were classified into high and low AC003986.3 expression subgroups. Survival curves of the high and low-expression subgroups were built and compared. Multivariate Cox regression was performed to explore the independent risk factors for patient survival. The result of multivariate Cox regression was demonstrated as a forest plot, and the death risks of all the patients were predicted by the forest plot. For further validation of the prediction model, patients were classified into high and low-risk subgroups according to the model-predicted death risks. Survival curves were built and compared between these subgroups. Discrimination capacity of AC003986.3 expression and the multivariate Cox regression prediction model was demonstrated by building the receiver operating characteristic curves (ROCs) for predicting 1, 2, 3, and 5-year survival, and areas under the curves (AUCs) were calculated.

Functional prediction

The target of AC003986.3 was predicted with Ensemble Browser (http://asia.ensembl.org/index.html). GSEA was performed to explore AC003986.3 related gene sets enrichment in Gene Ontology (GO) and Kyoto Encyclopedia of Genes and Genomes (KEGG) pathways with the software of GSEA (version 4.0.3, https://www.gsea-msigdb.org/gsea/downloads.jsp). The number of permutations was set to 1000 and the metric for ranking genes was set to Singal2Noise. For KEGG pathways, the results were filtered by $p$ value $<5 \%$ and normalized enrichment score $>1$ and ranked by increasing q-value. For GO pathways, the results were filtered by $p$-value $<1 \%$ and normalized enrichment score $>1$.

\section{Statistical analysis}

Figure plotting and statistical analysis were done with the R software (version 3.6.2, https://www.rproject.org/). Spearman linear regression was adopted to explore the Correlation between gene expression and age. Wilcoxon rank-sum test was used to compare gene expression between males and females. Kruskal-Wallis rank-sum test was used to compare the gene expression profiles among different tumor grade and race subgroups. Multivariate Cox regression was used to screen the risk factors for patient survival and determine the weights of the variates in the prediction model. The log-rank test was used to compare the differences between survival curves. Packages used in this study for figure plotting and statistical analysis mainly include ggplot2, survival, survminer, Hmisc, and survivalROC.

\section{Results}

AC003986.3 expression is positively correlated with patient age and pathological grade 
Correlation between AC003986.3 expression and clinical features of patient age, race, and gender and glioma pathological grade were presented in Fig. 1. AC003986.3 expression increases with patient age ( $P$ $<0.001)$, and the correlation coefficient was $0.22(95 \% \mathrm{Cl} 0.146-0.292)$ (Fig. 1A). The AC003986.3 expression did not differ between males and females ( 0.169 vs $0.155, P=0.488$, Fig. 1B), but increased positively with tumor grade $(P<0.001$, Fig. $1 C)$, with grade IV gliomas having the highest AC003986.3 expression. Similar to gender, the AC003986.3 expression was not different among races of the patient $(P=0.174)$. These results demonstrate that AC003986.3 expression was related to patient age and tumor pathological grade.

AC003986.3 expression profile is an independent risk factor for patient survival

Univariate Cox regression demonstrates that AC003986.3 expression was closely related to patient survival (Hazard Ratio (HR) $=4.710,95 \% \mathrm{Cl} 3.315-6.691, P<0.001)$. As Fig. 2 showed, patients with high AC003986.3 expression suffered a poor survival than those with low expression $(P<0.001)$. When the AC003986.3 expression profile was taken into multivariate Cox regression with gender, age, pathological grade, and race, we found age, pathological grade and AC003986.3 expression were independent risk factors for patient survival (Fig. 3). Age was a risk factor for patient survival (HR $=1.047,95 \% \mathrm{Cl} 1.035-$ $1.059, P<0.001)$. Referenced with grade II gliomas, grade III and grade IV glioma patients suffered a shorter survival $(\mathrm{HR}=2.540,95 \mathrm{Cl} 1.662-3.881, P<0.001$ for grade III gliomas, and $\mathrm{HR}=8.554,95 \% \mathrm{Cl}$ 5.280-13.57, $P<0.001$ for grade IV gliomas). Furthermore, the AC003986.3 expression profile was an independent risk factor for patient survival $(\mathrm{HR}=1.975,95 \% \mathrm{Cl} 1.312-2.966, P=0.001)$.

Survival curves were built and compared between the model-predicted high and low death risk subgroups (Fig. 4). As the figure showed, the high-risk subgroups harbored a poorer survival than the low-risk subgroup $(P<0.001)$. Discrimination capacity of AC003986.3 and the multivariate Cox regression model was shown in Fig. 5. With only AC003986.3 expression, the accuracy for predicting 1, 2, 3, and 5-year patient survival reached $0.774,0.729,0.728$ and 0.703 , respectively (Fig. 5A). The model demonstrated relatively high accuracy in predicting 1, 2, 3 and 5 -year survival of the patients (AUC $=0.864,0.899,0.912$, and 0.897, respectively, Fig. 5B).

AC00393986.3 interacts with TWIST1 and is mainly involved in tumor cell metabolism

Target prediction with the Ensemble Browser revealed that this novel IncRNA may interact with TWIST1 to regulate its degradation and function (Fig. 6A). KEGG enrichment analysis revealed nine enriched pathways (Fig. 6B). They were: Biosynthesis of unsaturated fatty acids, Butanone metabolism, Fatty acid metabolism, Glycolysis gluconeogenesis, Lysine degradation, Pentose phosphate pathway, Peroxisome, Propanoate metabolism, and valine leucine and isoleucine degradation. As to GO enrichment, as filtered by $\mathrm{p}$-value $<1 \%, 21$ pathways were identified. The top 10 enriched pathways in high expression group and the 6 enriched pathways in the low expression group were demonstrated in Fig. 6C.They were: $\mathrm{C} 21$ steroid hormone biosynthetic process, Detection of temperature stimulus, Establishment of epithelial cell polarity, Indole containing compound metabolic process, microbody, microbody lumen, microbody part, monocarboxylic acid catabolic process, negative regulation of macroautophagy, Organic acid catabolic 
process, Oxidoreductase activity acting on the aldehyde or oxo group of donors and or NADP as acceptor, Pericardium development, Regulation of synaptic transmission dopaminergic, response to lead ion, sensory perception of temperature stimulus. KEGG and GO enrichment analysis demonstrate that AC00393986.3 may be a key regulator for substance and energy metabolism, which is important to sustain the hypermetabolism of tumor cells.

\section{Discussion}

In this study, we found that AC00393986.3 expression was positively related to patient age and pathological grade in gliomas, and was an independent risk factor for patient survival. Functional prediction revealed that this gene might play important roles in regulating tumor cell metabolism through TWIST1. These results demonstrated that AC003986.3 was a potential target for gene therapy in gliomas.

Despite substantial progress in understanding the genetic mechanism in tumorigenesis, the treatment for gliomas remains difficult and challenging. One responsible reason is the high heterogeneity of the tumor. Further analysis of DNA, RNA, and proteins might further refine what should be targeted and lead to novel clinical trials.[8] Long noncoding RNAs (IncRNAs) are a variety of RNA transcripts longer than 200 nucleotides and lack of protein-encoding capacity.[9] Previously, IncRNAs were deemed as spurious wastes without function during transcription.[10] Recently, IncRNAs were recognized to play widespread roles in gene regulation and other cellular processes. [9] LncRNAs have also been implicated in cancer progressions, such as stemness, proliferation, angiogenesis and drug resistance.[11] Many IncRNAs have been identified as regulators in glioma progression. For example, HOXA11-AS could regulate cell cycle progress and was closely related to glioma grade and poor prognosis.[12] Similar functions were found with another IncRNA of HOTAIR.[13] In this study, we found the expression of the novel IncRNA AC003986.3 increased with of glioma malignancy $(P<0.001$, Fig. 1C), and AC003986.3 expression was an independent risk factor for predicting patient survival irrespective of patient age and tumor pathological grade $(\mathrm{HR}=1.975,95 \% \mathrm{Cl} 1.312-2.966, P=0.001$, Fig. 3$)$. Patients with higher AC003986.3 expression suffered a poorer survival than those with low expression $(P<0.001$, Fig. 2). Prediction accuracy with only AC003986.3 expression surpassed 0.70 for 1, 2, 3 and 5 years survivals (Fig. 5A), and the accuracy reached even higher (nearly 0.90) when combined with other clinical features (Fig. 5B). These results suggest that AC003986.3 may be a potential treatment target for gliomas.

The functions of AC003986.3 have not been reported. Interaction target prediction with Ensemble Browser revealed that this novel IncRNA might interact with TWIST1 and regulate its degradation and function. TWIST1 is a well-known regulator of epithelial-mesenchymal transition, tumorigenesis, and invasion in gliomas, [14-16] and is a predictor of prognosis for the patients. [17] Not only in gliomas, but in a variety of cancers such as breast, lung, prostate, gastric, bladder, pancreatic, and so on, TWIST1 was a key regulator in tumor initiation, progression, metastasis, apoptosis, the resistance of chemotherapy, and cancer stem cell formation.[18, 19] TWIST1 is also an important node in the intracellular signaling transduction network and linked to a variety of key signaling pathways such as FGF, TGF- $\beta$, NF-KB, and 
Notch, all of which were vital for both normal biological and pathological processes. $[19,20]$ All these results further confirmed the priceless potential of AC003986.3 for gene therapy.

KEGG gene set enrichment analysis revealed that AC003986.3 related genes were enriched in Biosynthesis of unsaturated fatty acids, Butanone metabolism, Fatty acid metabolism, Glycolysis gluconeogenesis, Lysine degradation, Pentose phosphate pathway, Peroxisome, Propanoate metabolism, and valine leucine and isoleucine degradation (Fig. 6B), demonstrating that AC003986.3 was mainly involved in cell energetic and synthetic metabolism. The tumor is hallmarked by reprogrammed pathways of nutrient acquisition and metabolism to meet the high demand for aggressive biological properties.[21] The reprogrammed metabolism activities always improved tumor cell adaption to provide a selective advantage during tumorigenesis.[21] Tumor cell metabolism targeted therapies have been reported both in animal models and clinical practice.[22, 23] Similar to KEGG analysis, GO enrichment analysis also indicated the potential role of AC003986.3 in tumor cell metabolism (Fig. 6C). These results suggest AC003986.3 may be a potential target for tumor metabolism-targeted treatment.

How AC003986.3 was involved in reprogrammed tumor metabolism was is unclear. As the interactor of AC003986.3, TWIST1 has been reported as a key regulator for metabolism.[24-27] Pan et al reported that TWIST1 was selectively expressed in adipose tissue, and was recruited to suppress mitochondrial metabolism and uncoupling, maintaining the of energy homeostasis.[24] Furthermore, TWIST1 was also reported to take part in glucose metabolism [26] and mitochondrial oxidative metabolism.[27] Along with our findings from the Ensemble Browser (Fig. 6A), we can infer that it is TWIST1 that mediates the regulation of AC003986.3 in glioma cell metabolism. Future experimental studies were needed to further elucidate the mechanism of how AC003986.3 regulates tumor cell metabolism and to validate the feasibility of utilizing AC003986.3 as the gene therapy target for gliomas.

\section{Limitations}

There are some limitations in this manuscript. First, other clinical features such as chemotherapy, radiology were not considered in this study, and these features would influence patient survival to some extent. Second, only bioinformatics analysis was conducted in this manuscript, molecular experiments were needed to further elucidate the function of AC003986.3.Third, only the TCGA dataset was analyzed in this manuscript, external validation was absent.

\section{Conclusions}

Although limitations, some conclusions still could be drawn from our study. The novel gene AC003986.3 is closely related to patient age and pathological grade in gliomas and is an independent risk factor for patient survival irrespective of age and pathological grade. Functional analysis reveals that this gene is mainly involved in reprogramming tumor cell metabolism, and this effect is probably TWIST1-dependent.

\section{Abbreviations}


AUC: area under the curve

GO: Gene Ontology

GSEA: gene set enrichment analysis

KEGG: Kyoto Encyclopedia of Genes and Genomes

ROC: receiver operating characteristic curve

TCGA: The Cancer Genome Atlas

\section{Declarations}

\section{Ethics approval and consent to participate}

Data used in this manuscript is publicly available from TCGA and consent to participate was not required. This study was approved by the Ethics Committee of Tian Tan Hospital.

\section{Consent for publication}

Not applicable.

\section{Availability of data and materials}

All the data used in this manuscript is obtained from TCGA.

\section{Competing interests}

The authors declare that they have no competing interests.

\section{Funding}

This study was supported by the China Postdoctoral Science Foundation (Grant No. 2019M660714).

\section{Authors' contributions}

QL designed this study and wrote the manuscript. HG, PL contributed to statistical analysis of the study. PJ designed and supervised the study. All authors read and approved the final manuscript. 


\section{Acknowledgements}

Not applicable.

\section{References}

1. Ostrom QT, Gittleman H, Liao P, Vecchione-Koval T, Wolinsky Y, Kruchko C, Barnholtz-Sloan JS: CBTRUS Statistical Report: Primary brain and other central nervous system tumors diagnosed in the United States in 2010-2014. Neuro-oncology 2017, 19(suppl_5):v1-v88.

2. Lapointe S, Perry A, Butowski NA: Primary brain tumours in adults. Lancet 2018, 392(10145):432446.

3. Louis DN, Perry A, Reifenberger G, von Deimling A, Figarella-Branger D, Cavenee WK, Ohgaki H, Wiestler OD, Kleihues P, Ellison DW: The 2016 World Health Organization Classification of Tumors of the Central Nervous System: a summary. Acta neuropathologica 2016, 131(6):803-820.

4. Zhu C, Zou C, Guan G, Guo Q, Yan Z, Liu T, Shen S, Xu X, Chen C, Lin Z et al: Development and validation of an interferon signature predicting prognosis and treatment response for glioblastoma. Oncoimmunology 2019, 8(9):e1621677.

5. Guo XX, Su J, He XF: A 4-gene panel predicting the survival of patients with glioblastoma. Journal of cellular biochemistry 2019, 120(9):16037-16043.

6. Wang $H, X u H, X u T$, Tan C, Jiang M, Chen Y, Hu X, Zhou J, Shen J, Qin R et al: High expression of TIG3 predicts poor survival in patients with primary glioblastoma. Tumour biology : the journal of the International Society for Oncodevelopmental Biology and Medicine 2017, 39(6):1010428317712135.

7. The TCGA Legacy. Cell 2018, 173(2):281-282.

8. Lapointe S, Perry A, Butowski NA: Primary brain tumours in adults. The Lancet 2018, 392(10145):432-446.

9. Ulitsky I, Bartel DP: lincRNAs: genomics, evolution, and mechanisms. Cell 2013, 154(1):26-46.

10. Struhl K: Transcriptional noise and the fidelity of initiation by RNA polymerase II. Nature structural \& molecular biology 2007, 14(2):103-105.

11. Peng Z, Liu C, Wu M: New insights into long noncoding RNAs and their roles in glioma. Molecular cancer 2018, 17(1):61.

12. Wang Q, Zhang J, Liu Y, Zhang W, Zhou J, Duan R, Pu P, Kang C, Han L: A novel cell cycle-associated IncRNA, HOXA11-AS, is transcribed from the 5-prime end of the HOXA transcript and is a biomarker of progression in glioma. Cancer letters 2016, 373(2):251-259.

13. Zhang JX, Han L, Bao ZS, Wang YY, Chen LY, Yan W, Yu SZ, Pu PY, Liu N, You YP et al: HOTAIR, a cell cycle-associated long noncoding RNA and a strong predictor of survival, is preferentially expressed in classical and mesenchymal glioma. Neuro-oncology 2013, 15(12):1595-1603.

14. Mikheeva SA, Mikheev AM, Petit A, Beyer R, Oxford RG, Khorasani L, Maxwell JP, Glackin CA, Wakimoto $\mathrm{H}$, Gonzalez-Herrero I et al: TWIST1 promotes invasion through mesenchymal change in 
human glioblastoma. Molecular cancer 2010, 9:194.

15. Mikheev AM, Mikheeva SA, Severs LJ, Funk CC, Huang L, McFaline-Figueroa JL, Schwensen J, Trapnell C, Price ND, Wong $S$ et al: Targeting TWIST1 through loss of function inhibits tumorigenicity of human glioblastoma. Molecular oncology 2018, 12(7):1188-1202.

16. Mikheeva SA, Camp ND, Huang L, Jain A, Jung SY, Avci NG, Tokita M, Wolf-Yadlin A, Zhang J, Tapscott SJ et al: TWIST1 Heterodimerization with E12 Requires Coordinated Protein Phosphorylation to Regulate Periostin Expression. Cancers 2019, 11(9).

17. Nordfors K, Haapasalo J, Makela K, Granberg KJ, Nykter M, Korja M, Paavonen T, Haapasalo H, Soini $\mathrm{Y}$ : Twist predicts poor outcome of patients with astrocytic glioma. Journal of clinical pathology 2015, 68(11):905-912.

18. Zhao Z, Rahman MA, Chen ZG, Shin DM: Multiple biological functions of Twist1 in various cancers. Oncotarget 2017, 8(12):20380-20393.

19. Qin Q, Xu Y, He T, Qin C, Xu J: Normal and disease-related biological functions of Twist1 and underlying molecular mechanisms. Cell research 2012, 22(1):90-106.

20. Franco HL, Casasnovas J, Rodriguez-Medina JR, Cadilla CL: Redundant or separate entities?-roles of Twist1 and Twist2 as molecular switches during gene transcription. Nucleic acids research 2011, 39(4):1177-1186.

21. DeBerardinis RJ, Chandel NS: Fundamentals of cancer metabolism. Science advances 2016, 2(5):e1600200.

22. Patra KC, Wang Q, Bhaskar PT, Miller L, Wang Z, Wheaton W, Chandel N, Laakso M, Muller WJ, Allen $E L$ et al: Hexokinase 2 is required for tumor initiation and maintenance and its systemic deletion is therapeutic in mouse models of cancer. Cancer cell 2013, 24(2):213-228.

23. Clavell LA, Gelber RD, Cohen HJ, Hitchcock-Bryan S, Cassady JR, Tarbell NJ, Blattner SR, Tantravahi $R$, Leavitt $P$, Sallan SE: Four-agent induction and intensive asparaginase therapy for treatment of childhood acute lymphoblastic leukemia. The New England journal of medicine 1986, 315(11):657663.

24. Pan $D$, Fujimoto $M$, Lopes $A$, Wang YX: Twist-1 is a PPARdelta-inducible, negative-feedback regulator of PGC-1alpha in brown fat metabolism. Cell 2009, 137(1):73-86.

25. Jun HJ, Gettys TW, Chang JS: Transcriptional Activity of PGC-1alpha and NT-PGC-1alpha Is Differentially Regulated by Twist-1 in Brown Fat Metabolism. PPAR research 2012, 2012:320454.

26. Wang XX, Yin GQ, Zhang ZH, Rong ZH, Wang ZY, Du DD, Wang YD, Gao RX, Xian GZ: TWIST1 transcriptionally regulates glycolytic genes to promote the Warburg metabolism in pancreatic cancer. Experimental cell research 2020, 386(1):111713.

27. Fan J, Fan Y, Wang X, Niu L, Duan L, Yang J, Li L, Gao Y, Wu X, Luo C: PLCepsilon regulates prostate cancer mitochondrial oxidative metabolism and migration via upregulation of Twist1. Journal of experimental \& clinical cancer research : CR 2019, 38(1):337.

\section{Figures}


A

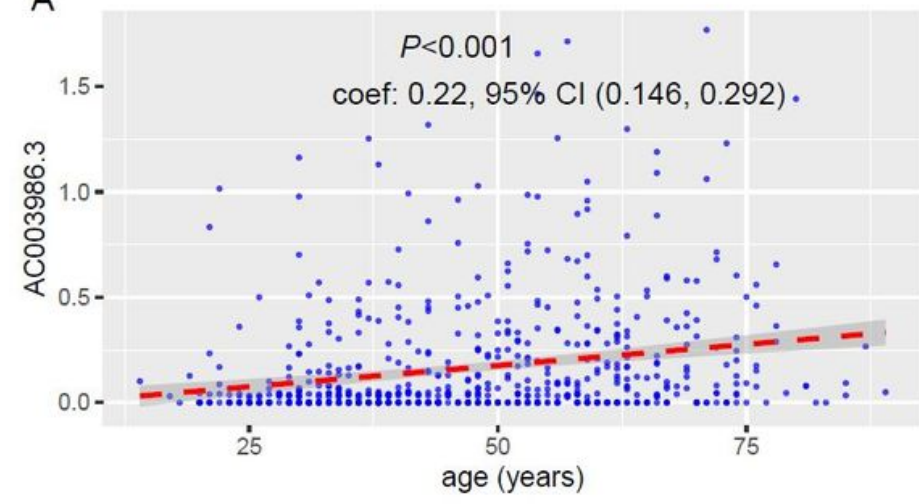

C

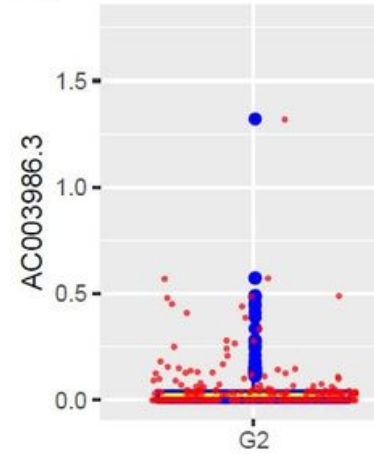

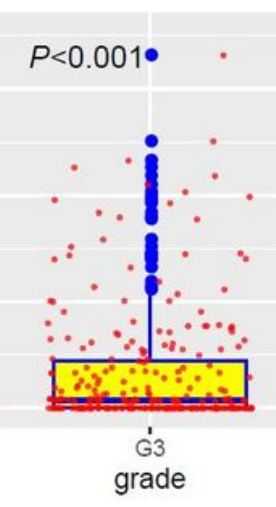

$B$

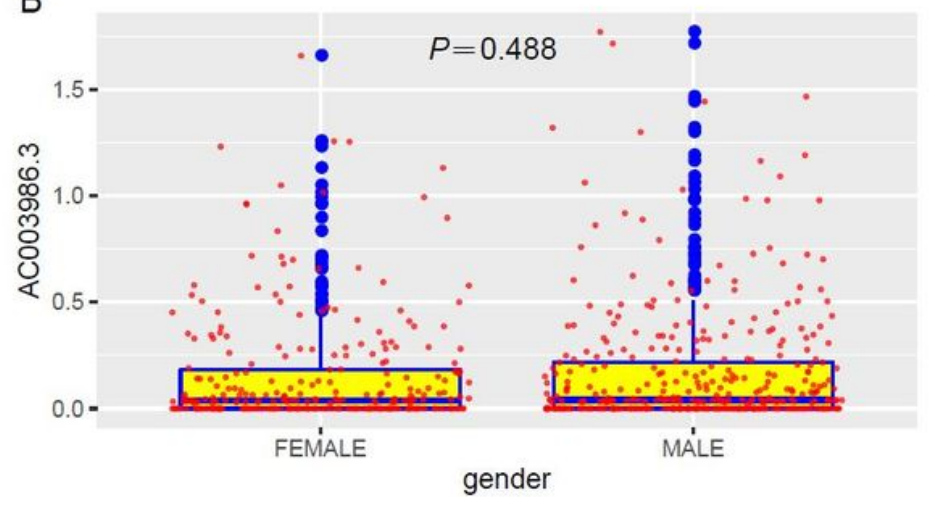

D

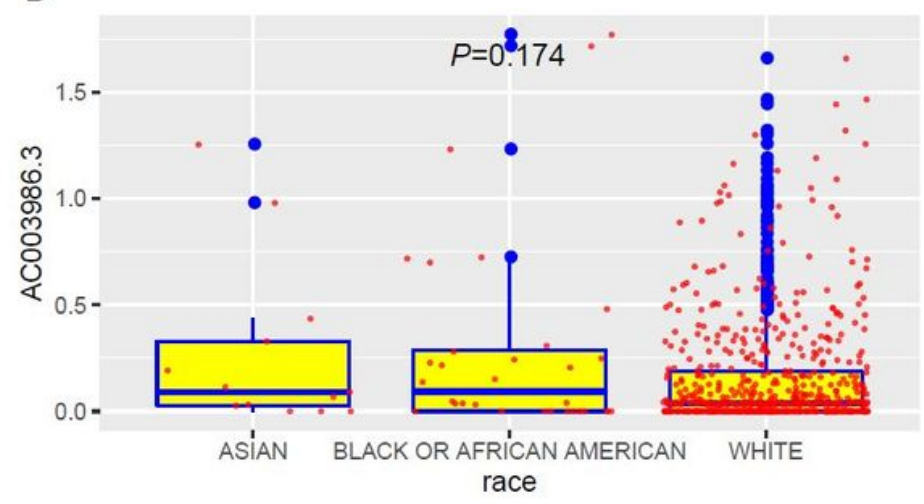

Figure 1

Correlation of AC003896.3 expression with patient age, gender, and race and tumor pathological grade. A: AC003986.3 was positively linear correlated to patient grade, with a correlation coefficient was 0.22 ( $95 \%$ $\mathrm{Cl} 0.146-0.292, \mathrm{P}<0.001)$. B: The expression of AC003896.3 was equally distributed between females and males. C: AC003986.3 expression increases with the tumor grade, with grade IV gliomas having the highest expression $(P<0.001)$. D: AC003986.3 expression was not influenced by patient race $(P=0.174)$. 


\section{Strata + genegroup=high + genegroup=low}

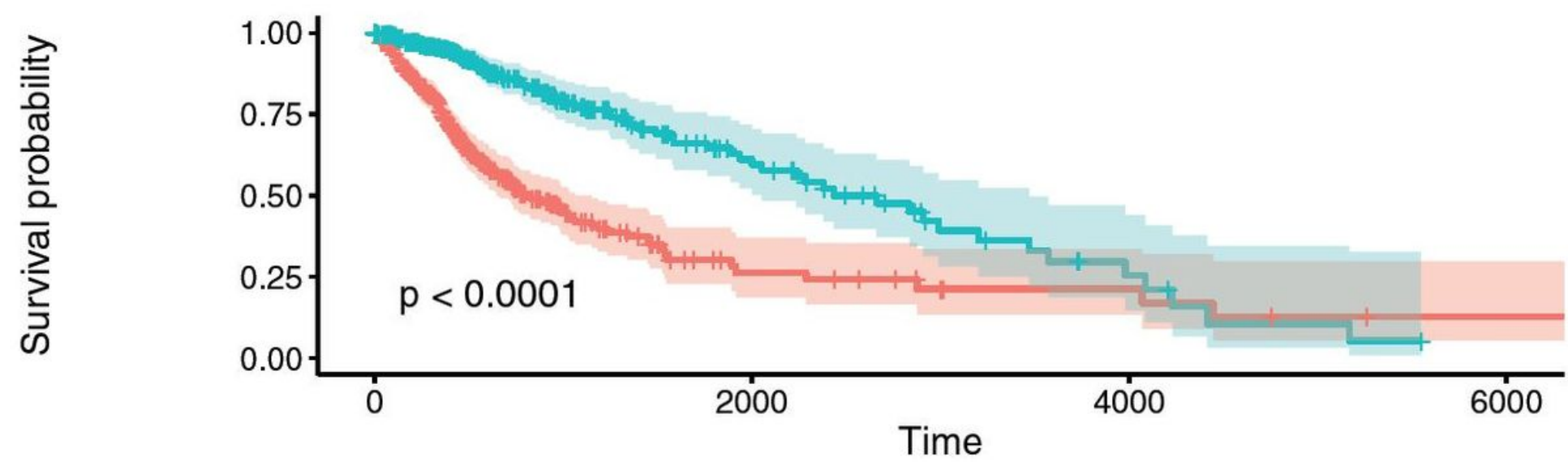

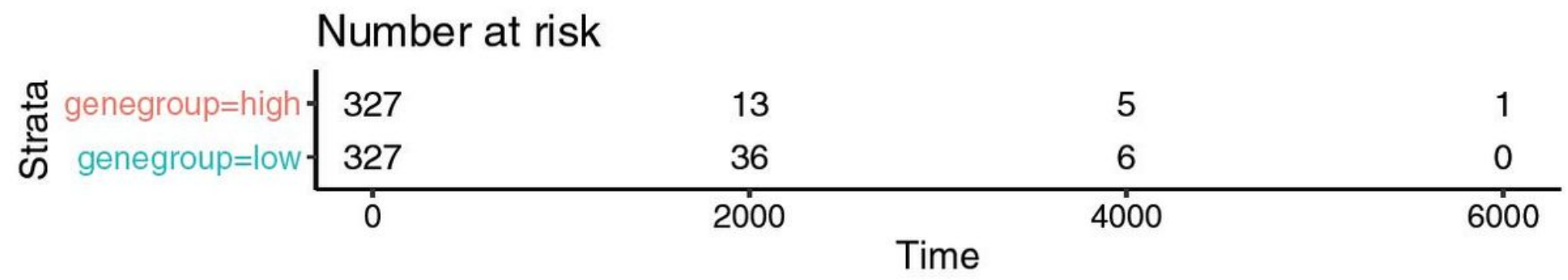

Cumulative number of events

\begin{tabular}{|c|c|c|c|c|}
\hline \multirow{3}{*}{ 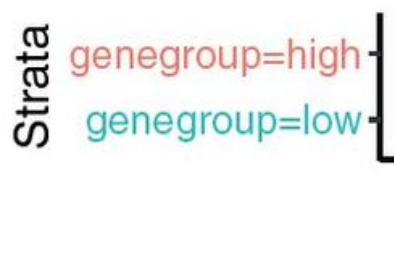 } & 0 & 142 & 144 & $\begin{array}{l}146 \\
7 ?\end{array}$ \\
\hline & 0 & 55 & 68 & 72 \\
\hline & 0 & 2000 & 4000 & 6000 \\
\hline
\end{tabular}

Figure 2

Patient survival of low and high AC003986.3 expression subgroups. Patients with high AC003986.3 expression suffered a poorer survival than those with low expression (log-rank test, $\mathrm{P}<0.001$ ). 


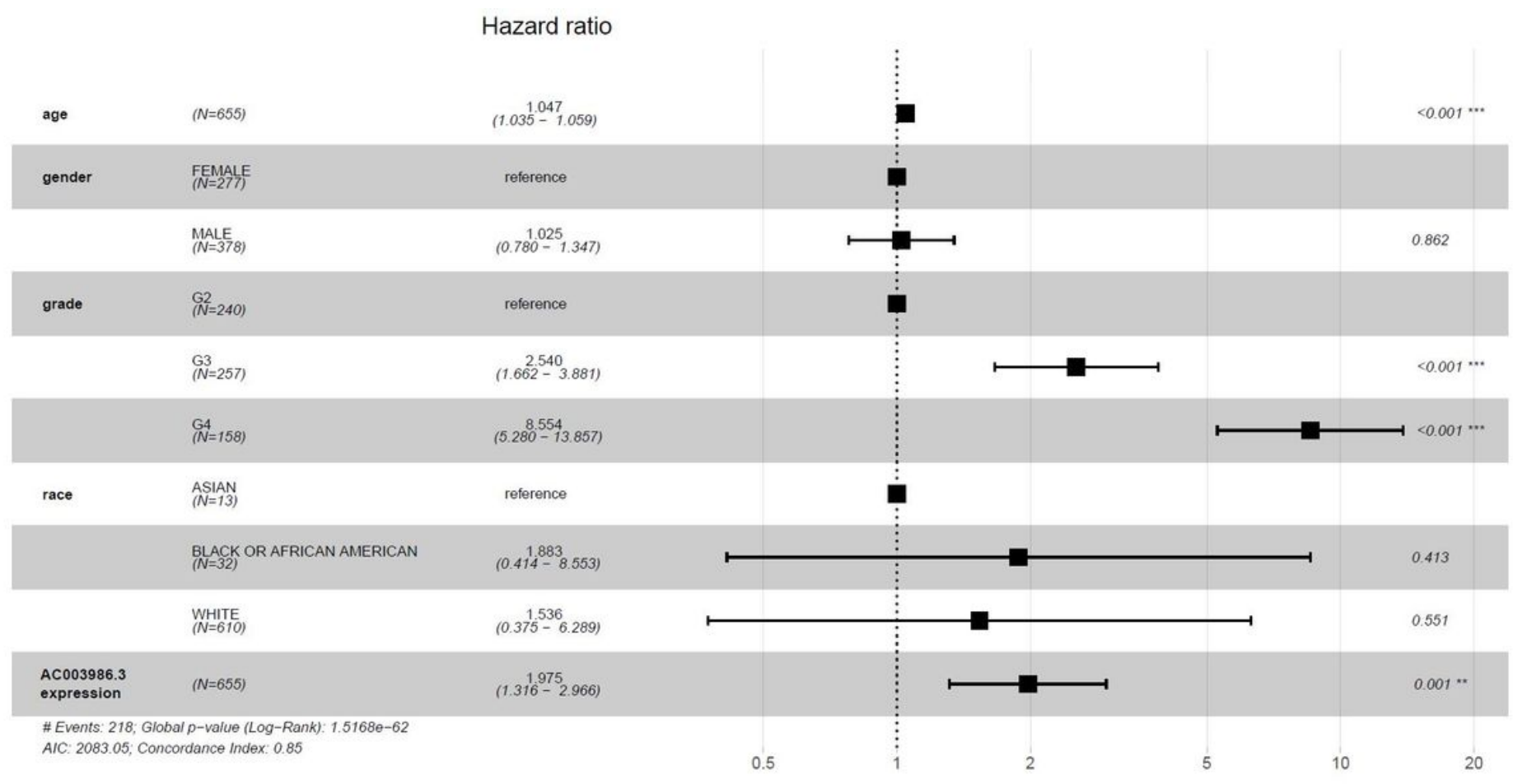

\section{Figure 3}

Forest plot of the multivariate Cox regression for patient survival. Patient age (HR=1.047, 95\% $\mathrm{Cl} 1.035$ $1.059, \mathrm{P}<0.001$ ), pathological grade (referenced by grade2, $\mathrm{HR}=2.540,95 \mathrm{Cl} 1.662-3.881, \mathrm{P}<0.001$ for grade III gliomas, and $\mathrm{HR}=8.554,95 \% \mathrm{Cl} 5.280-13.57, \mathrm{P}<0.001$ for grade IV gliomas) and AC003986.3 expression $(\mathrm{HR}=1.975,95 \% \mathrm{Cl} 1.312-2.966, \mathrm{P}=0.001)$ were independent risk factors for glioma patient survivals. 


\section{Strata + riskgroup=high + riskgroup=low}

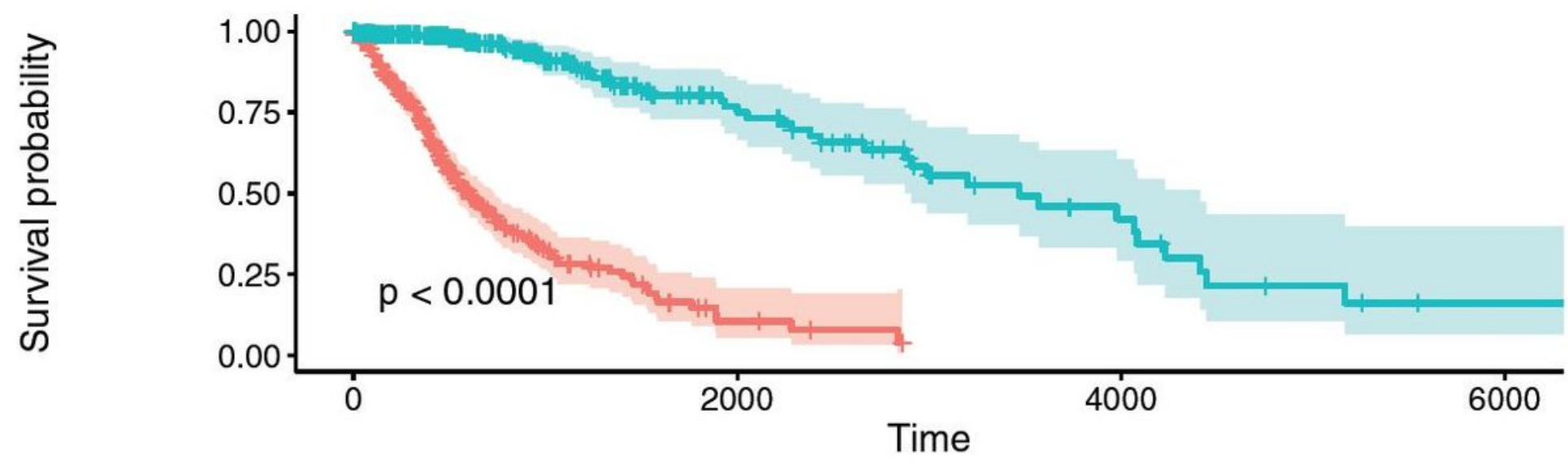

\begin{tabular}{|c|c|c|c|c|}
\hline \multirow{3}{*}{ 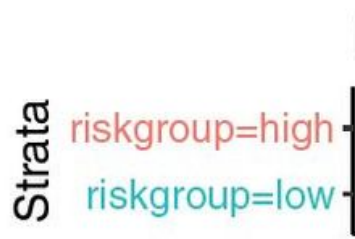 } & \multicolumn{4}{|c|}{ Number at risk } \\
\hline & 327 & 5 & 0 & 0 \\
\hline & 327 & 44 & 11 & 1 \\
\hline & 0 & 2000 & 4000 & 6000 \\
\hline
\end{tabular}

Cumulative number of events

\begin{tabular}{|c|c|c|c|c|}
\hline \multirow{3}{*}{ 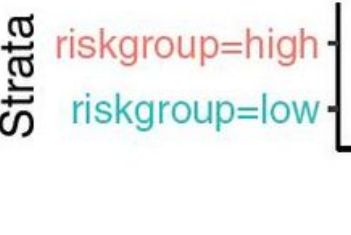 } & 0 & 170 & 172 & 172 \\
\hline & 0 & 27 & 40 & 46 \\
\hline & 0 & 2000 & 4000 & 6000 \\
\hline
\end{tabular}

\section{Figure 4}

Patient survival of low and high-risk subgroups. Patient death risk was calculated with the forest plot, and survival curves were compared between the high and low-risk groups. Patients with high death risk suffered a poorer survival than those with low risk (log-rank test, $\mathrm{P}<0.001)$. 
A

performance of AC003986.3 in predicting

$1,2,3$ and 5 year survival

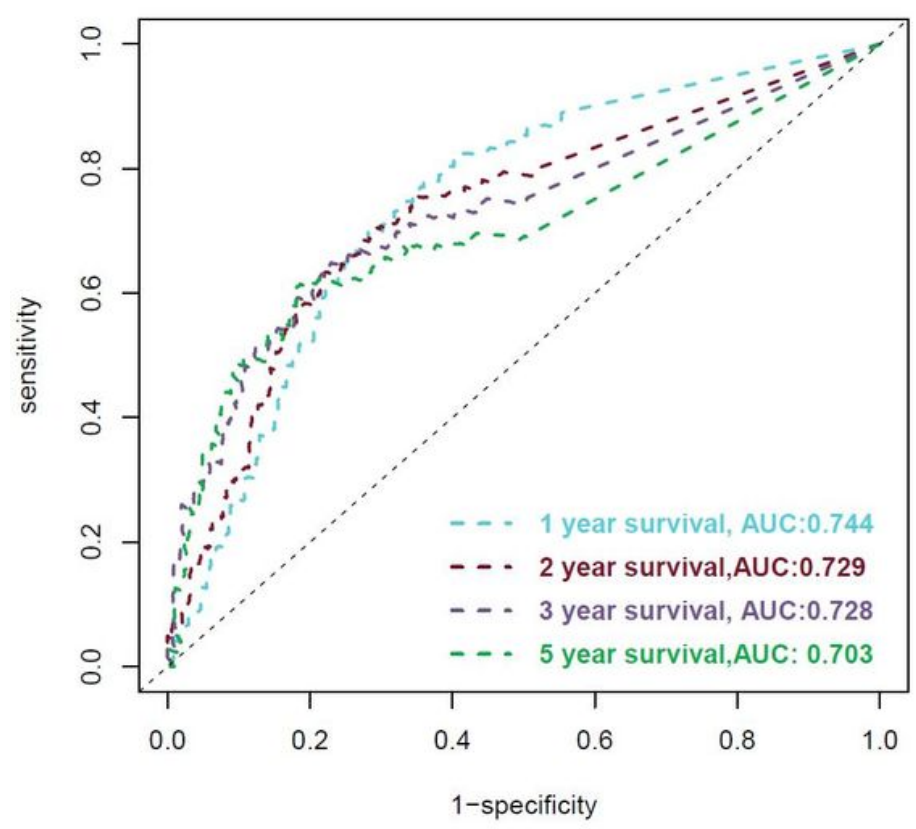

B performance of the model in predicting $1,2,3$ and 5 year survival

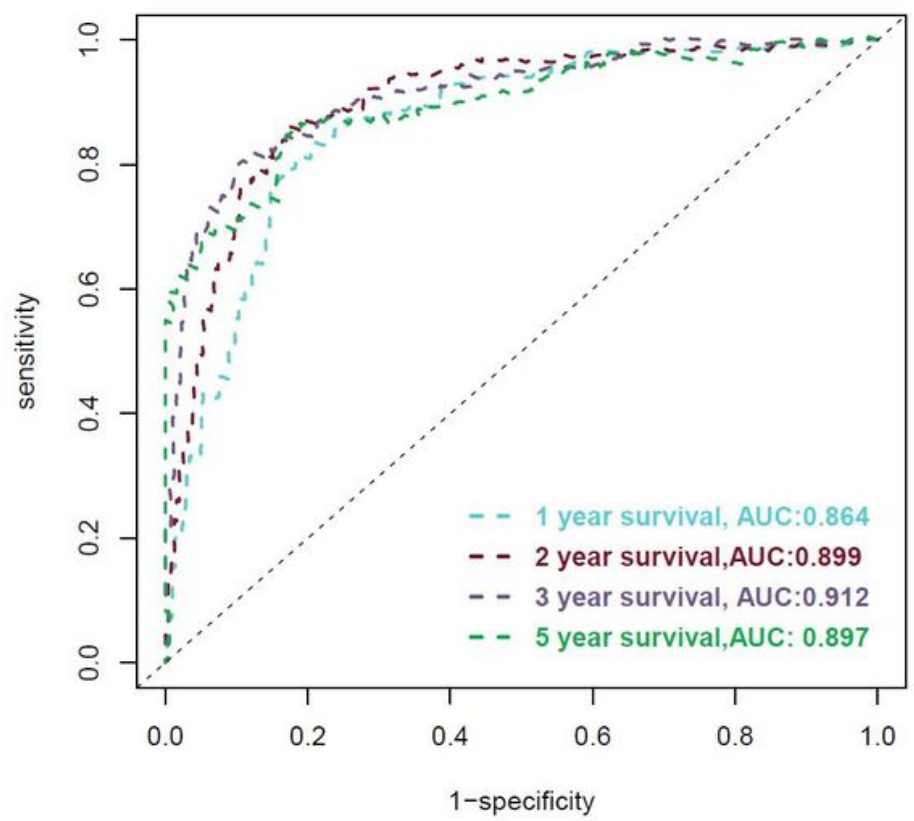

Figure 5

ROCs of AC003986.3 expression and forest plot model in predicting patient survival. A: Receiver operating characteristic curves were built and the accuracy of using AC003986.3 expression in predicting $1,2,3$, and 5 -year patient survival was $0.744,0.729,0.728$, and 0.703 , respectively. B: Receiver operating characteristic curves were built and the accuracy of using the forest plot model in predicting 1, 2, 3, and 5year patient survival was $0.864,0.899,0.912$, and 0.897 , respectively. ROC: Receiver operating characteristic curve. 


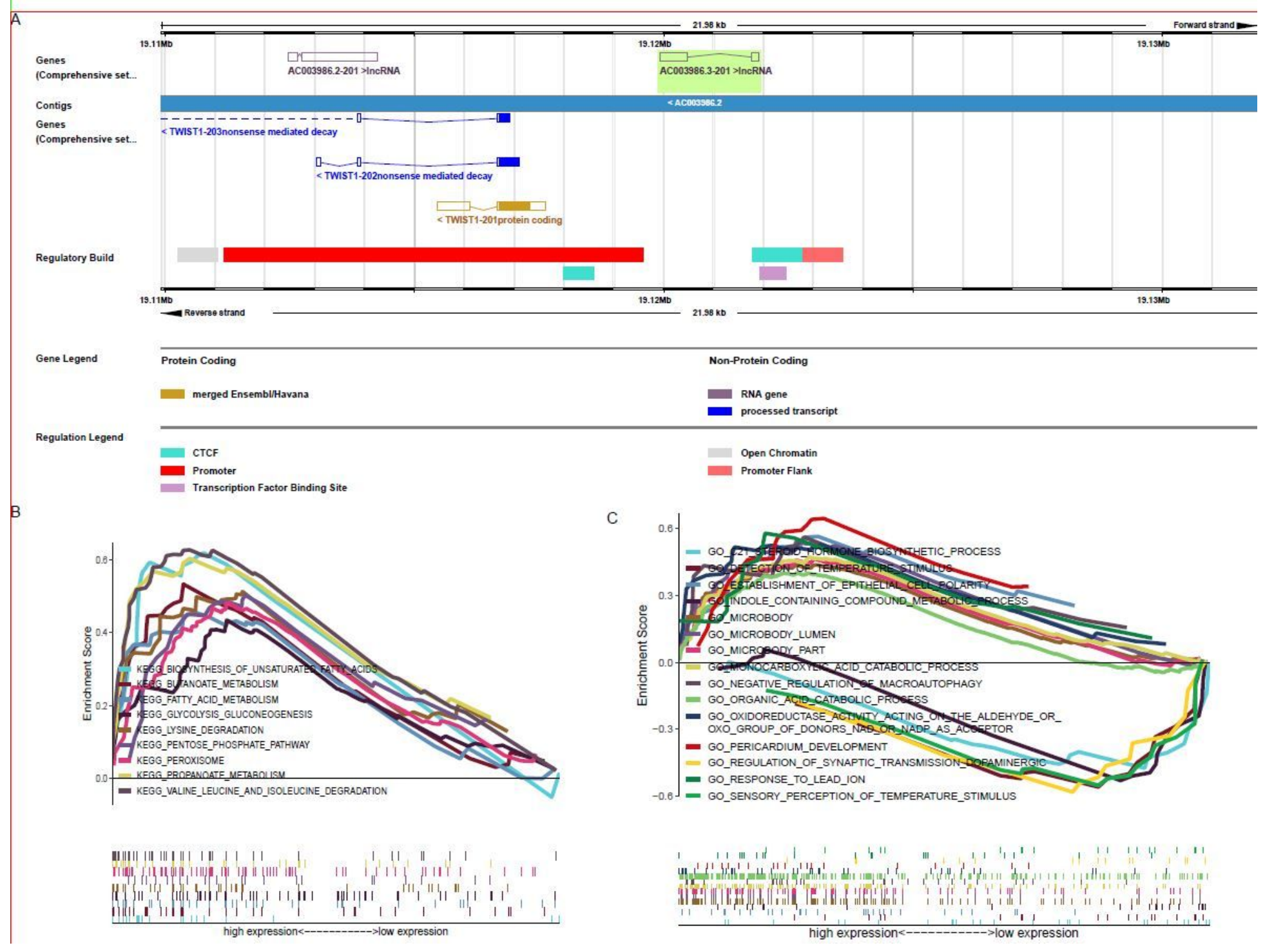

Figure 6

Functional prediction of AC003986.3. A: functional prediction of AC003986.3 with Ensemble Browser. AC003986.3 is a novel IncRNA and can regulate TWIST1 degradation through two nonsense mRNA decay locus. There is a binding site for AC003986.3 in TWIST1 protein. B: Gene set enrichment of AC003986.3 related genes in the KEGG pathways. Gene set enrichment analysis in KEGG pathways revealed that AC003986.3 related gene sets were enriched in Biosynthesis of unsaturated fatty acids, Butanone metabolism, Fatty acid metabolism, Glycolysis gluconeogenesis, Lysine degradation, Pentose phosphate pathway, Peroxisome, Propanoate metabolism, and valine leucine and isoleucine degradation. Filter criteria: p-value < 5 and normalized enrichment score >1. C: Gene set enrichment of AC003986.3 related genes in Gene Ontology. Gene set enrichment analysis Gene Ontology revealed that AC003986.3 related gene sets were mainly enriched in C21 steroid hormone biosynthetic process, Detection of temperature stimulus, Establishment of epithelial cell polarity, Indole containing compound metabolic process, microbody, microbody lumen, microbody part, monocarboxylic acid catabolic process, negative regulation of macroautophagy, Organic acid catabolic process, Oxidoreductase activity acting on the aldehyde or 
oxo group of donors and or NADP as acceptor, Pericardium development, Regulation of synaptic transmission dopaminergic, response to lead ion, sensory perception of temperature stimulus. KEGG: Kyoto Encyclopedia of Genes and Genomes, GO: gene ontology. 